OPEN ACCESS

Edited by: Antonio Politano,

Universitã degli Studi della Calabria, Italy

Reviewed by: Qihui Wu,

Quanzhou Normal University, China Arout Chelvane Jeyaramane, Defence Metallurgical Research Laboratory, India

*Correspondence: Manfred Albrecht manfred.albrecht@physik. uni-augsburg.de

tBirgit Hebler and Alexander Hassdenteufel contributed equally to this work

Specialty section This article was submitted to Thin Solid Films, a section of the journal Frontiers in Materials

Received: 08 December 2015 Accepted: 27 January 2016 Published: 16 February 2016

Citation:

Hebler B, Hassdenteufel A, Reinhardt $P$, Karl $H$ and Albrecht $M$ (2016) Ferrimagnetic Tb-Fe Alloy Thin

Films: Composition and Thickness Dependence of Magnetic Properties and All-Optical Switching. Front. Mater. 3:8. doi: 10.3389/fmats.2016.00008

\section{Ferrimagnetic Tb-Fe Alloy Thin Films: Composition and Thickness Dependence of Magnetic Properties and All-Optical Switching}

\author{
Birgit Hebler $^{1 \dagger}$, Alexander Hassdenteufel ${ }^{2 \dagger}$, Patrick Reinhardt ${ }^{2}$, Helmut Karl $^{1}$ and \\ Manfred Albrecht ${ }^{1 *}$
}

'Institute of Physics, University of Augsburg, Augsburg, Germany, ${ }^{2}$ Institute of Physics, Technische Universität Chemnitz, Chemnitz, Germany

Ferrimagnetic rare earth - transition metal Tb-Fe alloy thin films exhibit a variety of different magnetic properties, which depend strongly on composition and temperature. In this study, first the influence of the film thickness $(5-85 \mathrm{~nm})$ on the sample magnetic properties was investigated in a wide composition range between 15 and 38 at.\% of Tb. From our results, we find that the compensation point, remanent magnetization, and magnetic anisotropy of the Tb-Fe films depend not only on the composition but also on the thickness of the magnetic film up to a critical thickness of about 20-30 nm. Beyond this critical thickness, only slight changes in magnetic properties are observed. This behavior can be attributed to a growth-induced modification of the microstructure of the amorphous films, which affects the short range order. As a result, a more collinear alignment of the distributed magnetic moments of Tb along the out-of-plane direction with film thickness is obtained. This increasing contribution of the Tb sublattice magnetization to the total sample magnetization is equivalent to a sample becoming richer in $\mathrm{Tb}$ and can be referred to as an "effective" composition. Furthermore, the possibility of all-optical switching, where the magnetization orientation of $\mathrm{Tb}-\mathrm{Fe}$ can be reversed solely by circularly polarized laser pulses, was analyzed for a broad range of compositions and film thicknesses and correlated to the underlying magnetic properties.

Keywords: ferrimagnetic alloy, fanning cone, layer thickness, rare earth - transition metal, sperimagnetism, Tb-Fe, all-optical switching

\section{INTRODUCTION}

Ferrimagnetic amorphous rare earth (RE)-transition metal (TM) Tb-Fe alloy thin films gained considerable attention in the past as a potential medium for thermomagnetic recording (Mimura et al., 1976a,b; Mansuripur, 1986; Hansen, 1987; Jamet et al., 1996; Suits et al., 1998) due to its low Curie temperature (Mimura et al., 1976a; Iijima et al., 1989) and tunable magnetic properties by varying composition (Mimura et al., 1976a, 1978; Satoh et al., 1985; Hassdenteufel et al., 2013). Today's significance lies primarily in the field of exchange-biased heterostructures (Radu et al., 2012; Romer et al., 2012; Schubert et al., 2013; Tang et al., 2015), giving rise to giant exchange bias fields, and all-optical switching (AOS) (Stanciu et al., 2007; Hassdenteufel et al., 2013), where the 
orientation of the magnetization can be reversed by circularly polarized femtosecond laser pulses without any external magnetic field. This ability depends strongly on the material properties and underlying microstructure; thus its characterization and their dependence on growth conditions, film thickness, and composition is of great importance for the understanding of the underlying mechanism.

In this regard, a lot of effort has been made to investigate the intrinsic magnetic properties over a wide composition as well as temperature range, and how they are influenced by the growth conditions, like sputtering pressure (Jiang et al., 2010), substrate choice (Na et al., 2003), substrate bias voltage (Alperin et al., 1975), or external strain (Ohta et al., 2003). These research activities were encouraged by improved preparation techniques, which allowed preparing thin films in the thickness range of only some nanometers at reproducible high quality. However, more than 40 years ago, Rhyne et al. (1972) published already the first results on amorphous $\mathrm{TbFe}_{2}$ bulk systems and shortly thereafter Mimura and Imamura (1976) reported on the properties of 900-nm-thick Tb-Fe films over a composition range between 10 and 50 at.\% of Tb. At 21 at.\% of Tb, the so-called room temperature compensation point is reached where the net magnetization vanishes as both antiparallelly aligned magnetic sublattices of $\mathrm{Fe}$ and $\mathrm{Tb}$ compensate each other. This is accompanied by a drastic increase in coercivity $H_{\mathrm{C}}$. Below this compensation point, the $\mathrm{Tb}$ magnetization dominates while above this temperature the Fe magnetization is the leading one. The same behavior is also observed for other $\mathrm{Tb}-\mathrm{Fe}$ samples of same composition but varying film thickness. However, there is a distinct dissimilarity; the compensation point varies with film thickness. This is at first glance a rather unexpected result. Nakada et al. (1987) gave a first explanation of this effect and proposed the formation of boundary layers at the film-buffer and film-capping interfaces. On the contrary, Malmhäll and Chen (1982) ascribed the thickness dependence to a microstructure-induced variation in the short range order during growth, which causes a change in the effective composition with increasing film thickness.

Another very important and rather surprising property of amorphous $\mathrm{Tb}-\mathrm{Fe}$ thin films is the presence of strong perpendicular magnetic anisotropy (PMA), which was observed for a rather wide composition range between 15 and 28 at.\% (Mimura and Imamura, 1976). This is mainly caused by a chemical anisotropy in the short range order of the atoms due to different atomic next neighbor distances and coordination numbers for $\mathrm{Tb}-\mathrm{Tb}$, $\mathrm{Fe}-\mathrm{Fe}$, and $\mathrm{Tb}-\mathrm{Fe}$ pairs in out-of-plane and in-plane direction (pair-order anisotropy). In the out-of-plane direction (growth direction), a preference exists for the $\mathrm{Tb}-\mathrm{Fe}$ near-neighbor pairing (Gambino and Cuomo, 1978; van Dover et al., 1985; Robinson et al., 1989; Tewes et al., 1991; Harris et al., 1992a,b; Hellman and Gyorgy, 1992; Hernando et al., 1996; Hufnagel et al., 1996), which can be induced by selective re-sputtering during the growth process (Gambino and Cuomo, 1978; Harris and Pokhil, 1999). This leads together with the interaction of the local crystal field with the $4 \mathrm{f} \mathrm{Tb}$ orbital, which has a disk-like ellipsoidal shape (magnetic moment pointing out perpendicular to the plane of the ellipsoid), via spin-orbit coupling to an outof-plane oriented sample magnetization (single ion anisotropy)
(Prados et al., 1997; Skomski and Sellmyer, 2009). Hence, the magnetic order in $\mathrm{Tb}-\mathrm{Fe}$ arises from the competition between the local magnetic anisotropies due to the local structural order and exchange interactions (Campbell, 1972; Rebouillat et al., 1977; Coey, 1978; Handrich and Kobe, 1980; Buschow, 1991; Duc, 1993; du Trémolet de Lacheisserie et al., 2005). This competition leads to a local distribution of the $\mathrm{Tb}$ and the $\mathrm{Fe}$ magnetic moments in the $\mathrm{Tb}$ and Fe sublattices (Coey, 1978), known as sperimagnetism (see Figure 1). For Fe moments, the angular distribution (fanning cone) in amorphous $\mathrm{Tb}-\mathrm{Fe}$ alloys was directly measured by Mössbauer spectroscopy using ${ }^{57} \mathrm{Fe}$ ions (Eymery et al., 1988; Rusakov, 1992; Johnson, 1996; Ruckert et al., 1997; Danh et al., 2000; Fleury-Frenette et al., 2001). Ruckert et al. determined the typical fanning cone angle of Fe between $20^{\circ}$ to $30^{\circ}$ (Ruckert et al., 1997). They additionally could show that the Fe fanning does not vary strongly with film thickness or temperature.

Besides these structural peculiarities such as short range order, leading to PMA in amorphous RE-TM ferrimagnets, researchers at the Radboud University in Nijmegen observed in 2007 an ultra-fast magnetization reversal process induced by circularly or linearly polarized laser pulses in amorphous ferrimagnetic GdFeCo alloy films (Stanciu et al., 2007; Vahaplar et al., 2009, 2012; Radu et al., 2011). With this so-called AOS, the orientation of a magnetic domain can be reversed by a femtosecond laser pulse without any external magnetic field (Stanciu et al., 2007). Thus, AOS may lead to technological breakthroughs by using polarized light for ultrafast magnetization manipulation for a variety of applications. Since this pioneering experimental discovery, AOS has also been demonstrated for a variety of other amorphous ferrimagnetic RE-TM alloys, such as TbFe (Hassdenteufel et al., 2013, 2014, 2015; Schubert et al., 2014), TbCo (Alebrand et al., 2012), TbFeCo (Finazzi et al., 2013), DyCo (Mangin et al., 2014), HoFeCo (Mangin et al., 2014), and just very recently for antiferromagnetically coupled heterostructures (Mangin et al., 2014; Schubert et al., 2014) as well as for ferromagnetic materials (Lambert et al., 2014). Considerable recent effort has been made
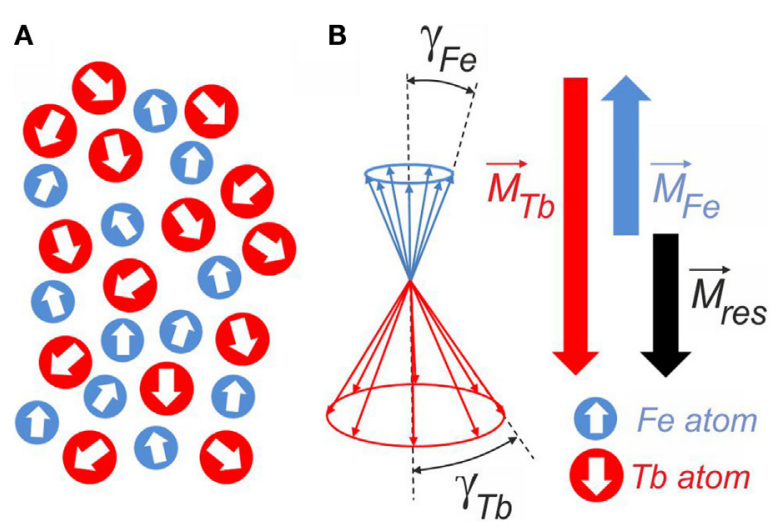

FIGURE 1 | (A) Magnetic moment distribution in an amorphous Tb-Fe material with two preferred antiparallelly oriented magnetic sublattices. (B) Averaged distribution of the magnetic moments (sperimagnetism). 
to investigate the physics of AOS and to reveal the underlying microscopic mechanisms. It was found that a rather small remanence magnetization $M_{\mathrm{R}}$ [ $<220 \mathrm{emu} / \mathrm{cm}^{3}$ (Hassdenteufel et al., 2015)] with strong temperature dependence (Atxitia et al., 2015) is an indispensable requirement for the AOS ability. In addition, different - sometimes very controversial - models have been proposed that particularly focused on the ferrimagnetic nature of the magnetic materials that show AOS and have been discussed along with recent experiments in Kirilyuk et al. (2013).

In this study, we first present a comprehensive investigation of the magnetic properties of $\mathrm{Tb}-\mathrm{Fe}$ thin films with thicknesses between 5 and $85 \mathrm{~nm}$ over a wide composition range between 15 and 38 at.\% of $\mathrm{Tb}$. It will be shown that in particular the compensation point and remanent magnetization of the $\mathrm{Tb}-\mathrm{Fe}$ samples depend not only on the composition but also on the thickness of the magnetic film. Thus, the ability of AOS was investigated and searched for correlations between AOS and the underlying magnetic properties of the samples.

\section{MATERIALS AND METHODS}

$\mathrm{Tb}-\mathrm{Fe}$ thin films were prepared under ultrahigh vacuum conditions by dc magnetron sputtering from element targets. The base pressure of the vacuum chamber was lower than $5 \times 10^{-8} \mathrm{mbar}$. As sputtering gas argon $(5 \mathrm{~N})$ with a partial pressure of $\mathrm{p}_{\mathrm{Ar}}=4.6 \mu \mathrm{bar}$ was used. The sputtering rate for each element was measured before the deposition by a quartz balance to adjust the electric power of the magnetron sputter gun and the sputtering time with respect to the desired film thickness and composition. For the co-sputtering of $\mathrm{Fe}$ and $\mathrm{Tb}$, the deposition rate of the $\mathrm{Tb}$ target was adjusted to values between 0.3 and $0.7 \AA / \mathrm{s}$, depending on the desired composition while the Fe rate was kept constant at $0.6 \AA / \mathrm{s}$. The deposition was carried out at room temperature on $375-\mu$ m-thick p-Si(100) substrates (p/boron, 10-20 $\Omega$ ) with a 100 -nm-thick thermally oxidized $\mathrm{SiO}_{2}$ layer on top as well as on glass microscope slides. To ensure a homogeneous deposition, the substrate rotates during the sputtering process. Against oxidation, the magnetic film was sandwiched between 5-nm-thick Pt buffer and capping layers. The stoichiometry and thickness of the prepared samples were characterized by Rutherford backscattering spectrometry (RBS). The amorphous structure of the $\mathrm{Tb}-\mathrm{Fe}$ films was confirmed by x-ray diffraction (XRD) and transmission electron microscopy (TEM). Compositional variations with film thickness were investigated by dynamic secondary ion mass spectroscopy (SIMS) studies. The SIMS depth profiles were measure by sputtering with a $3 \mathrm{keV} \mathrm{Cs}{ }^{+}$primary ion beam and detection of ${ }^{56} \mathrm{Fe}^{133} \mathrm{Cs}^{+}$and ${ }^{159} \mathrm{~Tb}^{+}$secondary ions. The integral magnetic properties of the samples were characterized by superconducting quantum interference device - vibrating sample magnetometry (SQUID-VSM). Two kinds of measurements were performed: remanent magnetization versus temperature $\left(M_{\mathrm{R}}-T\right)$ and magnetization versus field $(M-H)$. For the $M_{\mathrm{R}}-T$ measurements, the following procedure was applied: first, the samples were saturated at room temperature in a perpendicular magnetic field of $70 \mathrm{kOe}$. Then the samples were cooled down to $4 \mathrm{~K}$ without applying an external field. Thereafter, the temperature was again ramped from
4 to $400 \mathrm{~K}$, and the remanent magnetization $M_{\mathrm{R}}$ perpendicular to the sample plane was recorded. In addition, $M-H$ loops were measured at room temperature for in-plane and out-of-plane magnetic field. To give an estimate of the uniaxial magnetic anisotropy the following equation was used, $\left(K_{\mathrm{u}}=1 / 2 H_{\mathrm{a}} M_{\mathrm{R}}+2 \pi\right.$ $M_{\mathrm{R}}{ }^{2}$ ), which is corrected for the shape anisotropy contribution. Here, the anisotropy field $H_{\mathrm{a}}$ was extracted from the hard axis (in-plane) loop. The evolution of the fanning cone angle of the $\mathrm{Tb}$ moments can be obtained by analyzing the magnetization reversal at high magnetic fields. In particular, for Tb-Fe alloys, where the $\mathrm{Tb}$ moment dominate the net magnetization, an additional reversible increase in magnetization at higher external fields is observed, as shown in Figure 2 for a 14-nm-thick $\mathrm{Tb}_{29} \mathrm{Fe}_{71}$ film. This increase can be attributed to a more collinear alignment (compression) of the distributed magnetic moments of $\mathrm{Tb}$ along the external field direction. In contrast, for Fe rich $\mathrm{Tb}-\mathrm{Fe}$ films where the Fe moment is dominating, no pronounced magnetic field dependent increase of the magnetization was observed due to the stronger TM-TM exchange coupling of Fe (Heiman, 1976). Please note that this approach does not allow determining the exact fanning cone angle of the Tb moments, as the Tb fanning cannot be fully compressed and the Fe cone will certainly expand at the same time, thus these results should be treated with care and serve only as a first indicator.

In order to investigate the AOS ability of $\mathrm{Tb}-\mathrm{Fe}$ thin films, we used a Faraday imaging setup, revealing the orientation of sample magnetization as black $(\mathrm{M}-)$ and gray $(\mathrm{M}+)$ contrast. A regenerative laser amplifier system with pulse repetition rate of $250 \mathrm{kHz}$, center wavelength of $800 \mathrm{~nm}$, and pulse duration at the sample position of $100 \mathrm{fs}$ is used to provide the switching pulses. The polarization of these pulses are altered by an achromatic quarter wave plate and attenuated by a combination of a zero order half wave plate and polarizer. A detailed description of the setup can be found in Hassdenteufel et al. (2013). The switching experiment was performed as follows: first, the sample is homogeneously magnetized in one direction, e.g., M-. Subsequently,

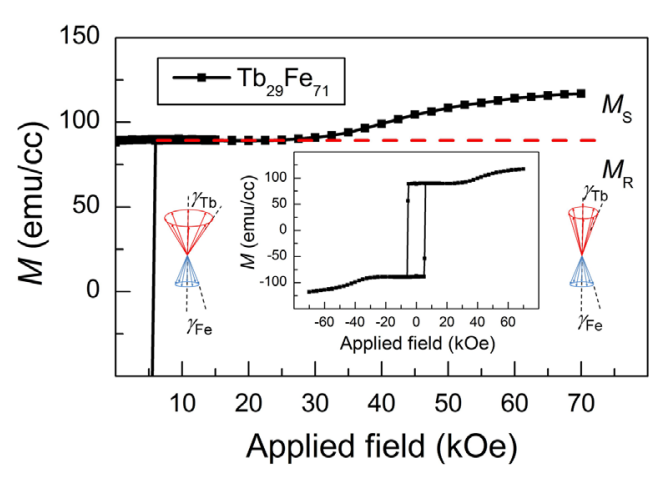

FIGURE 2 | Saturation branch of the hysteresis loop measured at room temperature by SQUID-VSM magnetometry for a 14-nm-thick $\mathbf{T b}_{29} \mathrm{Fe}_{71}$ film in out-of-plane geometry. Marked is the constant part of the hysteresis branch $M_{\mathrm{R}}$ before $M$ increases again due to the compression of the $\mathrm{Tb}$ fanning cone angle $\gamma_{\mathrm{Tb}}$ and reaches the saturation magnetization $M_{\mathrm{S}}$. Inset shows the full hysteresis loop. 
the sample is linearly moved below right-handed circularly polarized light $(\sigma+)$ at the switching threshold fluence. As far as the domain structure $(\mathrm{M}+)$ is written, the helicity is changed to the opposite direction (e.g., $\sigma-$ ). At the same fluence, the domain is again irradiated and, in the case of AOS, erased. This process of "writing" and "erasing" demonstrates unambiguously the AOS ability.

\section{RESULTS AND DISCUSSION}

\section{Composition and Thickness Dependence of Magnetic Properties of Tb-Fe Alloy Thin Films}

$M_{\mathrm{R}}-T$ curves are exemplarily plotted in Figures 3A,B for the 5- and 85-nm-thick $\mathrm{Tb}-\mathrm{Fe}$ film with varying $\mathrm{Tb}$ content between 15 and 35 at.\%, respectively. The typical transition from a Fe dominant to a Tb dominant film system with increasing $\mathrm{Tb}$ content accompanied by an increase in compensation temperature is observed. However, by analyzing the thickness dependence for a fixed alloy composition, we observed a similar shift of the $M_{\mathrm{R}}-T$ curves as shown exemplarily for $\mathrm{Tb}_{25} \mathrm{Fe}_{75}$ thin films in Figure 3C. By increasing the film thickness from 9 to $28 \mathrm{~nm}$, the compensation temperature shifts substantially from 150 to $370 \mathrm{~K}$, but remains rather unchanged for thicker films. At room temperature, we additionally measured the hysteresis $(M-H)$ loops of all Tb-Fe samples and extracted $M_{\mathrm{R}}$ and $H_{\mathrm{C}}$. The results are shown for the thickness series of $\mathrm{Tb}_{25} \mathrm{Fe}_{75}$ in Figure 4A. By increasing the thickness, the remanence decreases until reaching the compensation point at about $17 \mathrm{~nm}$. Afterwards, the $M_{\mathrm{R}}$ value is increasing up to a thickness of about $30 \mathrm{~nm}$ and does not change further. In contrast, the coercivity diverges to infinity close to the compensation thickness at room temperature, as expected. Furthermore, from the thickness dependent $M_{\mathrm{R}}-T$ curves, we selected the samples, which showed a compensation point at room temperature and plotted the corresponding composition $\left(\mathrm{Tb}_{\text {comp }}\right)$ as function of film thickness (Figure 4C). For increasing film thickness, the compensation of the magnetization occurs at lower Tb content and again remains rather unchanged beyond a thickness of about $30 \mathrm{~nm}$. Similar results were also reported by Malmhäll and Chen (1982), where a transition from a Fe dominant to a $\mathrm{Tb}$ dominant system at a critical film thickness of about 20-30 nm was found.

We can conclude that not only the film composition but also the film thickness influences substantially the remanent sample magnetization. This thickness dependence could be simply attributed to a variation in composition along the film thickness. However, this explanation was ruled out by analyzing the depth profiles of a 85-nm-thick $\mathrm{Tb}_{25} \mathrm{Fe}_{75}$ film extracted by SIMS. Here, a nearly constant $\mathrm{Tb}_{25} \mathrm{Fe}_{75}$ composition in the depth range between 10 and $80 \mathrm{~nm}$ has been found as shown in Figure 5. Close to the Pt seed and cover layer a strong matrix effect influencing drastically the Fe and $\mathrm{Tb}$ yield is observed, which does not allow drawing any conclusion about the $\mathrm{Tb}$ or Fe contents. However, in particular in the range of interest between 80 and $60 \mathrm{~nm}$, corresponding to the first $10-30 \mathrm{~nm}$ of
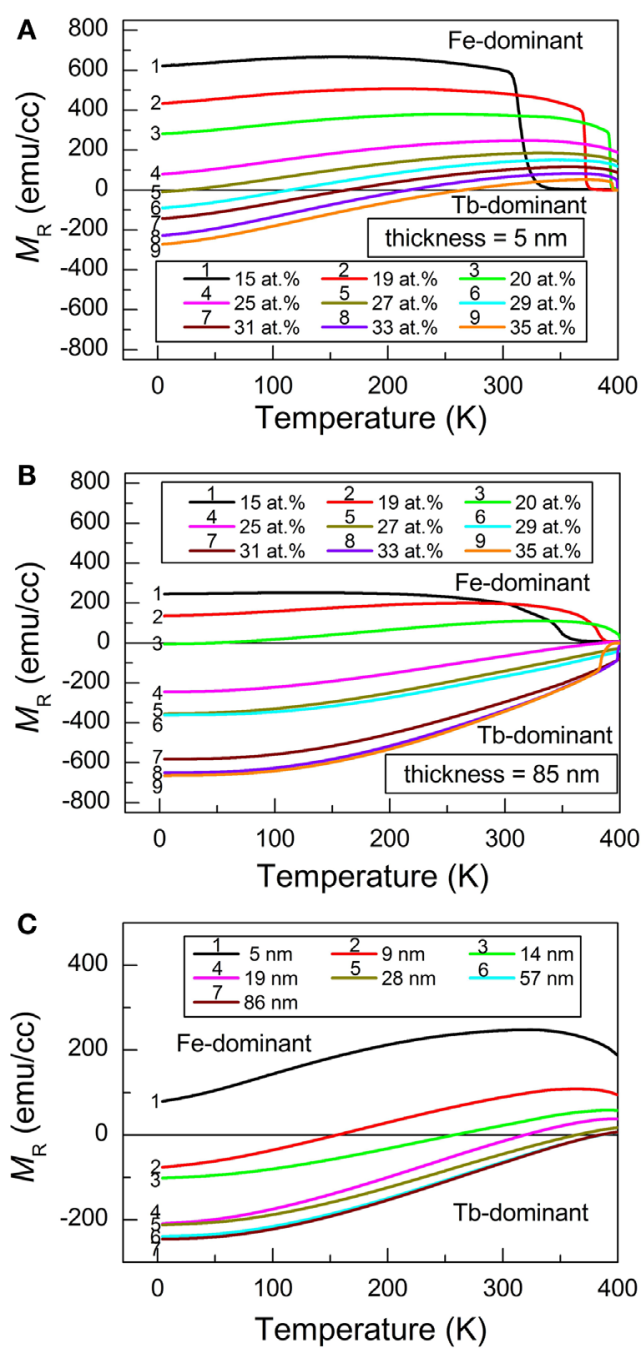

FIGURE 3 | Remanent sample magnetization $\boldsymbol{M}_{\mathrm{R}}$ versus temperature for (A) a 5-nm and (B) a 85-nm-thick $\mathrm{Tb}_{x} \mathrm{Fe}_{100-\mathrm{x}}$ film. (C) The remanent magnetization of the $\mathrm{Tb}_{25} \mathrm{Fe}_{75}$ film shifts from the Fe to the $\mathrm{Tb}$ dominated site with increasing film thickness.

the $\mathrm{Tb}-\mathrm{Fe}$ film from the substrate, no apparent difference in composition compared to the variation observed for thicker regions is observed. Thus, a systematic change in composition with thickness cannot account for the variation in magnetic properties. The same conclusion was also reported for similar films using electron microprobe analysis (Yuan et al., 1993) and by measuring the Curie temperature in dependence of film thickness (Malmhäll and Chen, 1982), which did not reveal a strong variation with thickness. Furthermore, an oxidation process of $\mathrm{Tb}$ next to the $\mathrm{SiO}_{2}$ substrate surface could be excluded as well (Malmhäll and Chen, 1982).

Thus, to answer the question of how the thickness dependence of $M_{\mathrm{R}}$ can be explained, we have to take the sperimagnetic nature of the amorphous films into account. Assuming that the fanning cone angle of Fe does not change much with film thickness (Ruckert et al., 1997), the behavior presented in Figure 4A can 

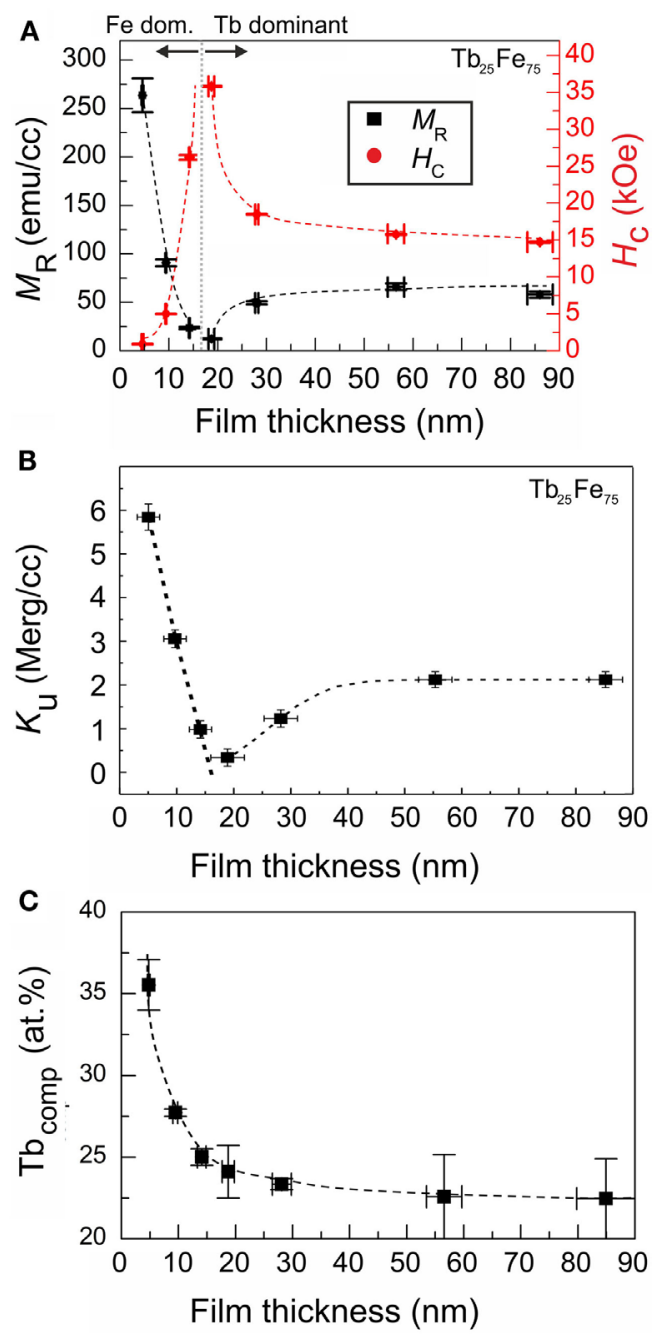

FIGURE 4 | (A) Remanent sample magnetization $M_{\mathrm{R}}$ and coercivity $H_{\mathrm{C}}$ for $\mathrm{Tb}_{25} \mathrm{Fe}_{75}$ films at room temperature for different film thicknesses. $M_{\mathrm{R}}\left(H_{\mathrm{C}}\right)$ decreases (increases) for increasing film thickness and reaches almost zero (infinity) at the room temperature compensation film thickness of about $17 \mathrm{~nm}$. For thicker films $M_{\mathrm{R}}\left(H_{\mathrm{C}}\right)$ increases (decreases) again and reaches a constant value for film thicknesses larger than $30 \mathrm{~nm}$. (B) Uniaxial magnetic anisotropy $K_{u}$ for $\mathrm{Tb}_{25} \mathrm{Fe}_{75}$ films at room temperature as function of film thickness. (C) Room temperature compensation composition $\mathrm{Tb}_{\text {comp }}$ versus magnetic film thickness. Lines are guide to the eyes.

be explained by an improved, more collinear alignment of the $\mathrm{Tb}$ moments along the out-of-plane direction with film thickness (reduction of the fanning cone angle). Such a compression of the fanning cone will result in an increased contribution of the $\mathrm{Tb}$ sublattice magnetization to the total sample magnetization along the film normal, which is equivalent to a sample becoming richer in $\mathrm{Tb}$ ["effective" composition (Malmhäll and Chen, 1982)]. From the observed reversible increase of the sample magnetization in the hysteresis loop toward saturation $\left(M_{S}\right)$ (see Figure 2), the $\left(M_{\mathrm{R}} / M_{\mathrm{S}}\right)$ ratio can be extracted. The ratio of $\left(M_{\mathrm{R}} / M_{\mathrm{S}}\right)$ is related to the fanning cone angle and decreases with increasing film thickness for $\mathrm{Tb}-\mathrm{Fe}$ films with a fixed $\mathrm{Tb}$

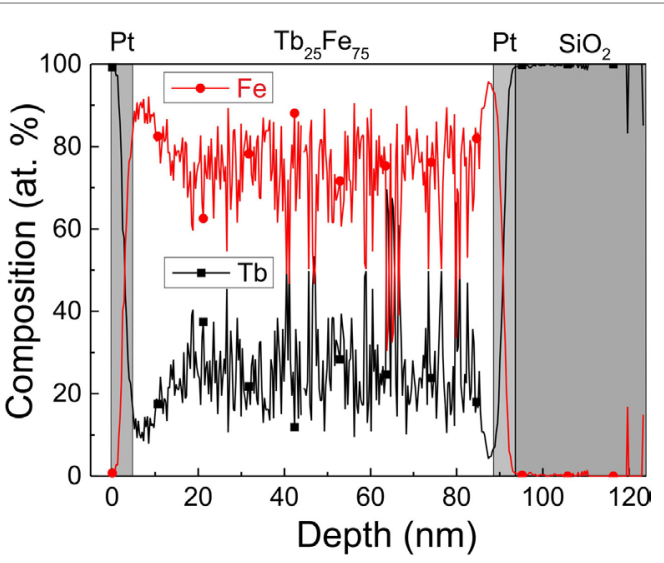

FIGURE 5 | SIMS composition depth profile of the following layer stack: $\mathrm{Pt}(5 \mathrm{~nm}) / \mathrm{Tb}_{25} \mathrm{Fe}_{75}(85 \mathrm{~nm}) / \mathrm{Pt}(5 \mathrm{~nm}) / \mathrm{SiO}_{2}(100 \mathrm{~nm}) / \mathrm{Si}(100)$ substrate.

content. But beyond a critical thickness of about $20-30 \mathrm{~nm}$, only a marginal change is observed. Thus, the compression of the fanning cone with thickness can explain the thickness dependence of the magnetic properties.

The origin for the changing distribution of the magnetic moments in the first $20-30 \mathrm{~nm}$ of the amorphous alloy has to be related to the morphology and microstructure including short range order. This is also supported by the similar thickness dependence of the uniaxial magnetic anisotropy $K_{\mathrm{u}}$ (Figure $4 \mathrm{~B}$ ). In this regard, the observed variation in magnetic anisotropy for film thickness up to $20-30 \mathrm{~nm}$ is directly associated with the outof-plane single ion anisotropy. This anisotropy is induced by short range order where the number of out-of-plane $\mathrm{Tb}-\mathrm{Fe}$ pairs is changing with thickness until a steady state is reached. However, stress-induced contributions can also play a decisive role in this thickness range (Takagi et al., 1979). It can be speculated that re-sputtering effects during film growth and substrate influence might be the origin.

We have seen that the compensation point, remanent magnetization, and magnetic anisotropy of the $\mathrm{Tb}-\mathrm{Fe}$ samples depend not only on the composition but also on the thickness of the magnetic film. In this regard, it was already mentioned that a low remanent magnetization is a necessary condition for the AOS ability (Hassdenteufel et al., 2015). Now, it will be interesting to see the AOS capability for this sample series and how it is correlated to the underlying magnetic properties.

\section{Composition and Thickness Dependence of All-Optical Switching of Tb-Fe Alloy} Thin Films

First, we have investigated a 19 -nm-thick $\mathrm{Tb}_{30} \mathrm{Fe}_{70}$ film, which is expected to show AOS as demonstrated in a previous study (Hassdenteufel et al., 2013). After magnetic saturation (M+, Figure 6A) in an external perpendicular magnetic field, the sample is moved linearly below left-handed polarized light $(\sigma-)$ with a fluence of $2 \mathrm{~mJ} / \mathrm{cm}^{2}$, where a black rectangular reverse 


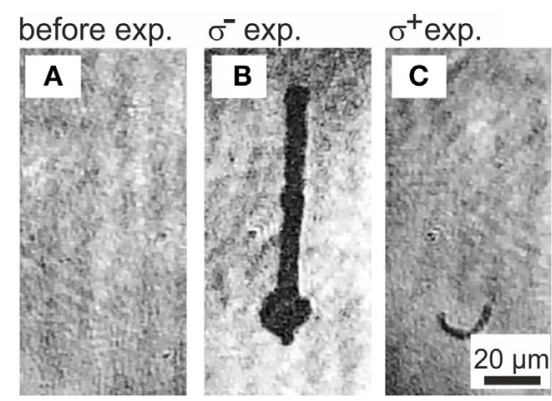

FIGURE 6 | All-optical helicity dependent magnetic switching in a 19-nm-thick $\mathrm{Tb}_{30} \mathrm{Fe}_{\mathbf{7 0}}$ film. (A) Faraday image of the homogeneously magnetized film before laser irradiation. (B) Faraday image showing a written stripe domain after interaction with right-handed circularly polarized laser light. (C) Erased stripe with left-handed circularly polarized laser light using the same fluence as in (B).

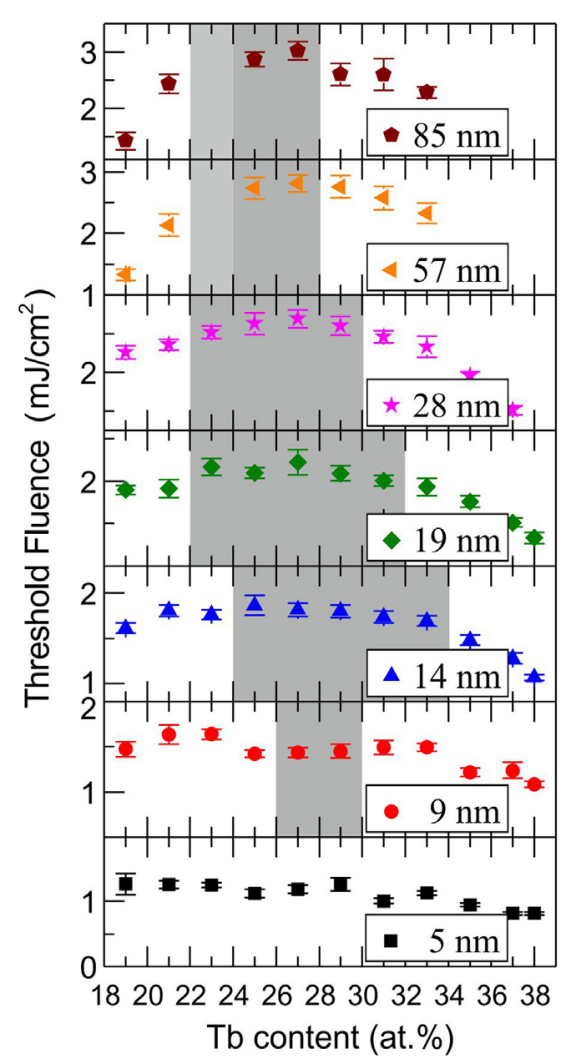

FIGURE 7 | Threshold fluence for all-optical switching (dark gray color) and otherwise pure thermal demagnetization (PTD) as function of Tb content and for various Tb-Fe film thicknesses. Please note that $\mathrm{Tb}_{23} \mathrm{Fe}_{77}$ samples with thickness of 57 and $85 \mathrm{~nm}$ were not investigated, however AOS is expected (light gray color).

domain structure $(\mathrm{M}-$ ) is indeed written (see Figure 6B). This $\mathrm{M}-$ domain is now switched back to a $\mathrm{M}+$ domain ("erased") by right-handed polarized light $(\sigma+)$ of the same fluence, as demonstrated in Figure 6C. In addition, we tried to reverse
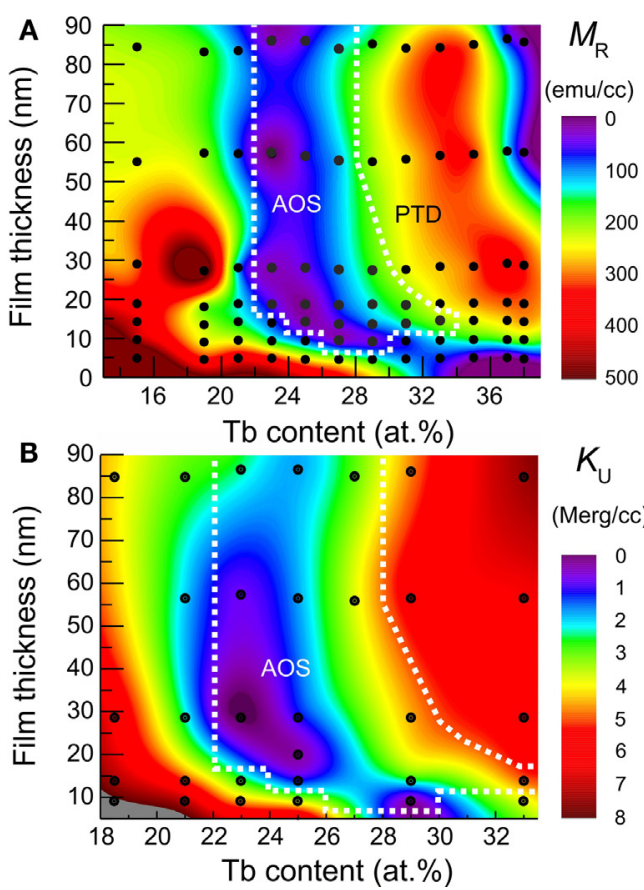

FIGURE 8 | (A) Remanent magnetization $M_{R}$ and (B) uniaxial magnetic anisotropy $K_{u}$ both at room temperature of Tb-Fe films of various thicknesses and composition. The dotted line indicates the area where AOS occurs.

with the same polarized light $(\sigma+)$ the surrounding $M+$ sample magnetization, which did not work. Thus, for this sample, AOS is confirmed, which occurs only for the correct combination of sample magnetization and circular polarization of light, i.e., $\mathrm{M}+(\mathrm{M}-)$ and $\sigma-(\sigma+)$. If the sample magnetization would also change for other combinations (e.g., $\mathrm{M}+$ and $\sigma+$ ), then a helicity independent thermal effect is obtained and the sample cannot be switched all-optically. In this case, the manipulation of the magnetization is governed by heat deposition into the material. This effect is named pure thermal demagnetization (PTD) (Hassdenteufel et al., 2013). AOS occurs only for specific $\mathrm{Tb}-\mathrm{Fe}$ alloy compositions and thicknesses as summarized in Figure 7. Only for a Tb content between 23 and 33 at.\% AOS is observed; however, this depends strongly on film thickness, starting at a minimum thickness of about $9 \mathrm{~nm}$ (only 27 and 29 at.\% Tb show AOS). With further increase in thickness, the composition range for AOS is increased revealing the largest range for a thickness of 14 and $19 \mathrm{~nm}$. For thicker films, the AOS range appears to be again reduced toward the Fe rich side. By comparing the corresponding values of the remanent magnetization and uniaxial magnetic anisotropy $K_{\mathrm{u}}$, which is summarized in Figure 8, it appears that the AOS ability is apparently correlated to a low remanence $\left(<220 \mathrm{emu} / \mathrm{cm}^{3}\right)$ with reduced magnetic anisotropy. However, these two quantities are related to each other and the low remanence is the relevant parameter as demonstrated for antiferromagnetically coupled Tb-Fe heterostructures (Schubert et al., 2014). Thus, this study further supports the importance of a low remanence as prerequisite for AOS (Hassdenteufel et al., 2015). 


\section{CONCLUSION}

In conclusion, we have prepared amorphous ferrimagnetic $\mathrm{Tb}-\mathrm{Fe}$ film samples over a wide composition range from $15 \mathrm{up}$ to 38 at.\% of $\mathrm{Tb}$ and with magnetic film thicknesses between 5 and $85 \mathrm{~nm}$. At a certain temperature, the compensation of the sample magnetization does not only depend on the composition but also on the specific thickness of the magnetic film. Growth-induced variations in the short range order resulting in a redistribution of the orientation of the $\mathrm{Tb}$ magnetic moments are responsible for the thickness dependence, while variations in composition with film thickness are insignificant. With increasing film thickness, the fanning cone angle of the $\mathrm{Tb}$ moments decreases, which causes an increasing contribution of the $\mathrm{Tb}$ sublattice magnetization to the total sample magnetization along the film normal. This behavior leads to a shift of the compensation temperature to higher temperatures with film thickness. Hence the magnetic properties of $\mathrm{Tb}-\mathrm{Fe}$ can be controlled not only by composition or temperature but also by film thickness, which has to be taken into account. In a further study, the AOS ability was investigated and correlated to the sample's magnetic properties. It was observed that AOS is connected to film samples, exhibiting low remanence $(<220 \mathrm{emu} /$ $\mathrm{cm}^{3}$ ), confirming the low remanence criterion. Furthermore,

\section{REFERENCES}

Alebrand, S., Gottwald, M., Hehn, M., Steil, D., Cinchetti, M., Laccour, D., et al. (2012). Light-induced magnetization reversal of high-anisotropy TbCo alloy films. Appl. Phys. Lett. 101:162408. doi:10.1063/1.4759109

Alperin, H. A., Cullen, J. R., and Clark, A. E. (1975). Magnetic properties of bulk amorphous $\mathrm{Tb}_{x} \mathrm{Fe}_{1-x}$. AIP Conf. Proc. 29:186. doi:10.1063/1.30576

Atxitia, U., Ostler, T. A., Chantrell, R. W., and Chubykalo-Fesenko, O. (2015). Optimal electron, phonon, and magnetic characteristics for low energy thermally induced magnetization switching. Appl. Phys. Lett. 107:192402. doi:10.1063/1.4935416

Buschow, K. H. J. (1991). Handbook of Magnetic Materials. North Holland: Elsevier.

Campbell, I. (1972). Indirect exchange for rare earths in metals. J. Phys. F Met. Phys. 47:3.

Coey, J. M. D. (1978). Amorphous magnetic order. J. Appl. Phys. 49:1646. doi: $10.1063 / 1.324880$

Danh, T. M., Duc, N. H., Thanh, H. N., and Teillet, J. (2000). Magnetic, Mössbauer and magnetostrictive studies of amorphous $\mathrm{Tb}\left(\mathrm{Fe}_{0.55} \mathrm{Co}_{0.45}\right)_{1.5}$ films. J. Appl. Phys. 87:7208. doi:10.1063/1.372970

du Trémolet de Lacheisserie, E., Gignoux, D., and Schlenker, M. (2005). Magnetism: Materials and Applications. New York: Springer Science+Business Media.

Duc, N. (1993). Effects of the 3d-5d hybridization on the $4 \mathrm{f}-3 \mathrm{~d}$ coupling in the rare earth-transition metal compounds. Phys. Status Solidi B 63, 63-66. doi:10.1002/ pssb.2221750231

Eymery, J., Fnidiki, A., Krishnan, R., Tessier, M., and Vitton, J. (1988). Conversionelectron Mössbauer spectroscopy studies in amorphous Tb-Fe films. Phys. Rev. B 38, 931-933. doi:10.1103/PhysRevB.38.11931

Finazzi, M., Savoini, M., Khorsand, A. R., Tsukamoto, A., Itoh, A., Dùo, L., et al. (2013). Laser-induced magnetic nanostructures with tunable topological properties. Phys. Rev.Lett. 110:177205. doi:10.1103/PhysRevLett.110.177205

Fleury-Frenette, K., Delwiche, J., Grandjean, F., Vandormael, D., and Long, G. J. (2001). Magnetic and conversion electron Mössbauer spectral study of amorphous thin films of $\mathrm{Dy}_{\mathrm{x}} \mathrm{Fe}_{100-\mathrm{x}}$ and $\mathrm{Dy}_{20} \mathrm{Fe}_{80-\mathrm{y}} \mathrm{Co}_{\mathrm{y}}$. J. Appl. Phys. 90:1934. doi:10.1063/1.1385574

Gambino, R. J., and Cuomo, J. J. (1978). Selective resputtering-induced anisotropy in amorphous films. J. Vac. Sci. Technol. 15:296. doi:10.1116/1.569574
Tb-Fe films as thin as $9 \mathrm{~nm}$ and as thick as $85 \mathrm{~nm}$ could be switched all-optically, which might be relevant for prospective magneto-optic or spintronic applications.

\section{AUTHOR CONTRIBUTIONS}

$\mathrm{BH}, \mathrm{AH}, \mathrm{HK}$, and $\mathrm{PR}$ prepared and characterized the thin film samples. $\mathrm{BH}, \mathrm{AH}$, and $\mathrm{PR}$ analyzed the magnetic data; $\mathrm{BH}, \mathrm{AH}$, and MA designed the research approach; $\mathrm{BH}, \mathrm{AH}$, and MA contributed to the development of the experiments and wrote the manuscript; all authors discussed the experiments and the manuscript.

\section{ACKNOWLEDGMENTS}

We thank C. Schubert (TU Chemnitz) for assistance in sample preparation, M. Daniel (TU Chemnitz) for RBS characterization and J. von Borany and R. Wilhelm for technical support at the RBS beam line at Helmholtz-Zentrum Dresden Rossendorf.

\section{FUNDING}

This research has received founding from the German Research Foundation (DFG, project number Al618/24-1).

Handrich, K., and Kobe, S. (1980). Amorphe Ferro- und Ferrimagnetika. Berlin: Akademie Verlag.

Hansen, P. (1987). Thermomagnetic switching in amorphous rare earth transition metal alloys. J. Appl. Phys. 62:216. doi:10.1063/1.339185

Harris, V., Aylesworth, K., Das, B., Elam, W., and Koon, N. (1992a). Structural origins of magnetic anisotropy in sputtered amorphous Tb-Fe films. Phys. Rev. Lett. 69, 1939-1942. doi:10.1103/PhysRevLett.69.1939

Harris, V. G., Elam, W. T., Koon, N. C., and Hellman, F. (1992b). Depositiontemperature dependence of structural anisotropy in amorphous Tb-Fe films. Phys. Rev. B 49:3637(R). doi:10.1103/PhysRevB.49.3637

Harris, V. G., and Pokhil, T. (1999). Evidence for selective resputtering as the growth mechanism of pair-order anisotropy in amorphous TbFe films. Bull. Mater. Sci. 22:503. doi:10.1007/BF02749962

Hassdenteufel, A., Hebler, B., Schubert, C., Liebig, A., Teich, M., Helm, M., et al. (2013). Thermally assisted all-optical helicity dependent magnetic switching in amorphous $\mathrm{Fe}_{100-x} \mathrm{~Tb}_{x}$ alloy films. Adv. Mater. Weinheim 25:3122. doi:10.1002/ adma.201300176

Hassdenteufel, A., Schmidt, J., Schubert, C., Hebler, B., Helm, M., Albrecht, M., et al. (2015). Low remanence criterion for helicity-dependent all-optical magnetic switching in ferrimagnets and heterostructures. Phys. Rev. B 91:104431. doi:10.1103/PhysRevB.91.104431

Hassdenteufel, A., Schubert, C., Schmidt, J., Richter, P., Zahn, D. R. T., Salvan, G., et al. (2014). Dependence of all-optical magnetic switching on the sublattice magnetization orientation in Tb-Fe thin films. Appl. Phys. Lett. 105:112403. doi:10.1063/1.4895933

Heiman, N. (1976). Modified mean field model for rare earth-iron amorphous alloys. J. Appl. Phys. 47:2634. doi:10.1063/1.322982

Hellman, F., and Gyorgy, E. M. (1992). Growth-induced magnetic anisotropy in amorphous Tb-Fe. Phys. Rev. Lett. 68:1391. doi:10.1103/ PhysRevLett.68.1391

Hernando, A., Prados, C., and Prieto, C. (1996). Anisotropy, magnetostriction and local chemical order in amorphous $\mathrm{Tb}_{\mathrm{x}} \mathrm{Fe}_{1-\mathrm{x}}(0.1<\mathrm{x}<0.55)$ thin films. J. Magn. Magn. Mater. 157/158:501. doi:10.1016/0304-8853(95)01124-2

Hufnagel, T., Brennan, S., Zschack, P., and Clemens, B. (1996). Structural anisotropy in amorphous Fe-Tb thin films. Phys. Rev. B 53:12024. doi:10.1103/ PhysRevB.53.12024 
Iijima, T., Ishii, O., and Hatakeyama, I. (1989). Magnetic and magnetooptic properties of In alloyed TbFe amorphous films. Appl. Phys. Lett. 54:2376. doi:10.1063/1.101086

Jamet, J., Meyer, P., Grolier, V., Ferré, J., and Houdy, P. (1996). Thermomagnetic writing and time resolved imaging in a $[\mathrm{Tb}(7.9 \AA) / \mathrm{Fe}(11 \AA)]_{20}$ multilayer. J. Magn. Soc. Jpn. 20, 217-222. doi:10.3379/jmsjmag.20.S1_217

Jiang, H. C., Zhang, W. L., Zhang, W. X., and Peng, B. (2010). Effects of argon pressure on magnetic properties and low-field magnetostriction of amorphous TbFe films. Physica B Condens. Matter 405, 834-838. doi:10.1016/j. physb.2009.07.130

Johnson, C. (1996). Characterization of magnetic materials by Mössbauer spectroscopy. J. Phys. D Appl. Phys. 59, 2266-2273. doi:10.1088/0022-3727/29/9/007

Kirilyuk, A., Kimel, A. V., and Rasing, T. H. (2013). Laser-induced magnetization dynamics and reversal in ferrimagnetic alloys. Rep. Prog. Phys. 76:026501. doi:10.1088/0034-4885/76/2/026501

Lambert, C.-H., Mangin, S., Varaprasad, B. S. D., Takahashi, Y. K., Hehn, M., Cinchetti, M., et al. (2014). All optical control of ferromagnetic thin films and nanostructures. Science 345:1337. doi:10.1126/science.1253493

Malmhäll, R., and Chen, T. (1982). Thickness dependence of magnetic hysteretic properties of $\mathrm{rf}$ sputtered amorphous $\mathrm{Tb}-\mathrm{Fe}$ alloy thin films. J. Appl. Phys. 53:7843. doi: $10.1063 / 1.330216$

Mangin, S., Gottwald, M., Lambert, C.-H., Steil, D., Uhlír, V., Pang, L., et al. (2014). Engineered materials for all-optical helicity-dependent magnetic switching. Nat. Mater. 13:286. doi:10.1038/nmat3864

Mansuripur, M. (1986). Magnetization reversal, coercivity, and the process of thermomagnetic recording in thin films of amorphous rare earth-transition metal alloys. J. Appl. Phys 61:1580. doi:10.1063/1.338094

Mimura, Y., and Imamura, N. (1976). Magnetic properties of amorphous TbFe thin films prepared by rf sputtering. Appl. Phys. Lett. 28:746. doi:10.1063/1.88638

Mimura, Y., Imamura, N., and Kobayashi, T. (1976a). Magnetic properties and curie point writing in amorphous metallic films. IEEE Trans. Magn. 12, 6-8. doi:10.1109/TMAG.1976.1059199

Mimura, Y., Imamura, N., and Kobayashi, T. (1976b). Curie point writing in amorphous magnetic films. Jpn. J. Appl. Phys. 15:933. doi:10.1143/JJAP.15.933

Mimura, Y., Imamura, N., Kobayashi, T., Okada, A., and Kushiro, Y. (1978). Magnetic properties of amorphous alloy films of Fe with Gd, Tb, Dy, Ho, or Er. J. Appl. Phys. 49:1208. doi:10.1063/1.325008

Na, S. M., Suh, S. J., and Lim, S. H. (2003). Fabrication condition effects on the magnetic and magnetostrictive properties of sputtered Tb-Fe thin films. J. Appl. Phys. 93:8507. doi:10.1063/1.1543870

Nakada, M., Toki, K., and Okada, M. (1987). Thickness dependence of effective composition for amorphous TbFe films. IEEE Trans. Magn. Jpn. 2, 348-349. doi:10.1109/TJMJ.1987.4549437

Ohta, M., Yamada, K., Satake, Y., Fujita, A., and Fukamichi, K. (2003). Origin of perpendicular magnetic anisotropy in Tb-Fe amorphous alloy. Mater. Trans. 44:2605-2610. doi:10.2320/matertrans.44.2605

Prados, C., Marinero, E., and Hernando, A. (1997). Magnetic interactions and anisotropy in amorphous TbFe films. J. Magn. Magn. Mater. 165:414. doi:10.1016/S0304-8853(96)00573-2

Radu, F., Abrudan, R., Radu, I., Schmitz, D., and Zabel, H. (2012). Perpendicular exchange bias in ferrimagnetic spin valves. Nat. Commun. 3:715. doi:10.1038/ ncomms 1728

Radu, I., Vahaplar, K., Stamm, C., Kachel, T., Pontius, N., Dürr, H. A., et al. (2011). Transient ferromagnetic-like state mediating ultrafast reversal of antiferromagnetically coupled spins. Nature 472:205. doi:10.1038/nature09901

Rebouillat, J. P., Lienard, A., Coey, J. M. D., Arrese-Boggiano, R., and Chappert, J. (1977). Magnetic structures and properties of the amorphous alloys $\mathrm{Dy}_{3}$; $\mathrm{T}$ $=$ Fe, Co, Ni. Physica B+C 86-88, 773-774. doi:10.1016/0378-4363(77)90680-5

Rhyne, J. J., Pickart, S. J., and Alperin, H. A. (1972). Direct observation of an amorphous spin-polarization distribution. Phys. Rev. Lett. 29:1562. doi:10.1103/ PhysRevLett.29.1562
Robinson, C. J., Samant, M. G., and Marinero, E. E. (1989). EXAFS study of the atomic structure of amorphous $\mathrm{Tb}_{20} \mathrm{Fe}_{80}$. Appl. Phys. A 49, 619-629. doi:10.1007/ BF00616986

Romer, S., Marioni, M. A., Thorwarth, K., Joshi, N. R., Corticelli, C. E., Hug, H. J., et al. (2012). Temperature dependence of large exchange-bias in TbFe-Co/Pt. Appl. Phys. Lett. 101:222404. doi:10.1063/1.4767142

Ruckert, T., Tappert, J., Brand, R. A., and Keune, W. (1997). Mössbauer-effect study of amorphous $\mathrm{Tb}_{1-\mathrm{x}} \mathrm{Fe}_{\mathrm{x}}$. J. Magn. Magn. Mater. 165, 411-413. doi:10.1016/ S0304-8853(96)00572-0

Rusakov, V. (1992). Mössbauer spectroscopy and magneto-optical studies of Tb-Fe films. IEEE Trans. Magn. 28, 2524-2526. doi:10.1109/20.179544

Satoh, T., Fukamichi, K., and Satoh, Y. (1985). Magnetic properties and thermal expansion of Fe-Tb amorphous alloys. IEEE Trans. Magn. Jpn. 1:796. doi:10.1109/TJMJ.1985.4548963

Schubert, C., Hassdenteufel, A., Matthes, P., Schmidt, J., Helm, M., Bratschitsch, R., et al. (2014). All-optical helicity dependent magnetic switching in an artificial zero moment magnet. Appl. Phys. Lett. 104:082406. doi:10.1063/1.4866803

Schubert, C., Hebler, B., Schletter, H., Liebig, A., Daniel, M., Abrudan, R., et al. (2013). Interfacial exchange coupling in $\mathrm{Fe}-\mathrm{Tb} /[\mathrm{Co} / \mathrm{Pt}]$ heterostructures. Phys. Rev. B 87:054415. doi:10.1103/PhysRevB.87.054415

Skomski, R., and Sellmyer, D. J. (2009). Anisotropy of rare-earth magnets. J. Rare Earths 27:675. doi:10.1016S1002/0721(08)60314-2

Stanciu, C. D., Hansteen, F., Kimel, A. V., Kirilyuk, A., Tsukamoto, A., Itoh, A., et al. (2007). All-optical magnetization recording with circularly polarized light. Phys. Rev. Lett. 99:047601. doi:10.1103/PhysRevLett.99.047601

Suits, J. C., Rugar, D., and Lin, C. J. (1998). Thermomagnetic writing in TbFe: modeling and comparison with experiment. J. Appl. Phys. 64:252. doi:10.1063/1.341474

Takagi, H., Tsunashima, S., Uchiyama, S., and Fujii, T. (1979). Stress induced anisotropy in amorphous Gd-Fe and Tb-Fe sputtered films. J. Appl. Phys. 50:1642. doi:10.1063/1.327223

Tang, M. H., Zhang, Z., Tian, S. Y., Wang, J., Ma, B., and Jin, Q. Y. (2015). Interfacial exchange coupling and magnetization reversal in perpendicular $[\mathrm{Co} / \mathrm{Ni}] \mathrm{N} /$ TbCo composite structures. Sci. Rep. 5:10863. doi:10.1038/srep 1086

Tewes, M., Zweck, J., and Hoffmann, H. (1991). Short range order in amorphous FeTb. J. Magn. Magn. Mater. 95, 43-48. doi:10.1016/0304-8853(91)90212-S

Vahaplar, K., Kalashnikova, A. M., Kimel, A. V., Gerlach, S., Hinzke, D., Nowak, U., et al. (2012). All-optical magnetization reversal by circularly polarized laser pulses: experiment and multiscale modeling. Phys. Rev. B 85:104402. doi:10.1103/PhysRevB.85.104402

Vahaplar, K., Kalashnikova, A. M., Kimel, A. V., Hinzke, D., Nowak, U., Chantrell, R., et al. (2009). Ultrafast path for optical magnetization reversal via a strongly nonequilibrium state. Phys. Rev. Lett. 103:117201. doi:10.1103/ PhysRevLett.103.117201

van Dover, R. B., Hong, M., Gyorgy, E. M., Dillon, J. F. Jr., and Albiston, S. D. (1985). Intrinsic anisotropy of Tb-Fe films prepared by magnetron Co sputtering. J. Appl. Phys. 57:3897. doi:10.1063/1.334908

Yuan, Y., Chevrier, F., Le Gall, H., Rommeluere, M., and Dumond, Y. (1993). Thickness dependence of magneto-optic effects in TbFeCo film. IEEE Trans. Magn. 29:3778. doi:10.1109/20.281297

Conflict of Interest Statement: The authors declare that the research was conducted in the absence of any commercial or financial relationships that could be construed as a potential conflict of interest.

Copyright (c) 2016 Hebler, Hassdenteufel, Reinhardt, Karl and Albrecht. This is an open-access article distributed under the terms of the Creative Commons Attribution License (CC BY). The use, distribution or reproduction in other forums is permitted, provided the original author(s) or licensor are credited and that the original publication in this journal is cited, in accordance with accepted academic practice. No use, distribution or reproduction is permitted which does not comply with these terms. 